\title{
Surgical outcome of a female patient with comorbid epilepsy and psychogenic non-epileptic seizures confirmed with video-EEG monitoring
}

\author{
Liang Qiao, Tao Yu, Yongjie Li \\ Beijing Institute of Functional Neurosurgery, Xuanwu Hospital of Capital Medical University, Beijing, China \\ Correspondence to: Liang Qiao. Beijing Institute of Functional Neurosurgery, Xuanwu Hospital of Capital Medical University, 45 Changchun Street, \\ Xicheng District, Beijing 100053, China. Email: qiaoliang79@aliyun.com.
}

\begin{abstract}
Despite common comorbidities of epileptic seizure (ES) and psychogenic nonepileptic seizure (PNES), reports regarding the clinical management of the two coexisting disorders have traditionally only focused on non-surgical approaches such as medication and psychotherapy. Epileptogenic zonectomy following comprehensive presurgical evaluation could lead to seizure control and even seizure free in refractory epilepsy patients. However, its effect on PNES which is of clinical significance remains to be explored. Here, we present a favorable surgical outcome in a middle-aged woman with refractory epilepsy with concomitant video-electroencephalograph-confirmed PNES. The patient has been ES free post rightsided anterior temporal lobectomy with unchanged antiepileptic medication. Meanwhile, remarkable improvement of her PNES has been reported although she did not undergo psychiatric or psychological therapy following surgery. The treatment success achieved in this case suggests that comorbid PNES should not deprive patients with intractable epilepsy patients a chance to undergo surgical intervention if their epileptogenic zones could be identified during presurgical evaluation. Some patients could obtain relieves of both ES and PNES from appropriately performed epilepsy surgery. Nevertheless, the mechanisms underlying the findings in this report are still unknown. Further study is needed to investigate whether epileptogenic zonectomy itself or the patient's positive mood brought by ES control contributes to postoperative alleviation of PNES.
\end{abstract}

Keywords: Surgery; comorbidity; psychogenic seizure; case report

Submitted Jul 20, 2020. Accepted for publication Dec 10, 2020.

doi: $10.21037 /$ atm-20-5423

View this article at: http://dx.doi.org/10.21037/atm-20-5423

\section{Introduction}

Psychogenic nonepileptic seizures (PNES) are episodes of unintentional movement and altered sensation or experience, usually triggered by stress (1). Despite possible behavioral similarity to epileptic seizures (ES), PNES is not associated with excessive synchronization of large neuronal populations generating ictal epileptiform discharges in the brain. PNES is commonly seen in epilepsy and neurology practice. It is estimated that the prevalence of PNES is about 2-33 per 100,000 (2). In clinical scenarios, PNES and ES can either develop in separate patient populations or co-exist in the same individuals. However, the prevalence of comorbidities of PNES and ES is still unknown. Studies have suggested that $50-60 \%$ of PNES patients have comorbid epilepsy (3), while $5.3-50 \%$ of epilepsy patients are reported to have confirmed PNES (4).

PNES is currently considered a conversion disorder with functional neurological symptoms according to the DSM-V (5) despite much debate about its classification and terminology $(6,7)$. Hence, thus far, clinical management of PNES is mostly by means of psychiatric and psychological therapies. Surprisingly, the treatment, particularly surgical attempts, for concomitant PNES and ES remains insufficiently explored. Herein, we report a case of video- 


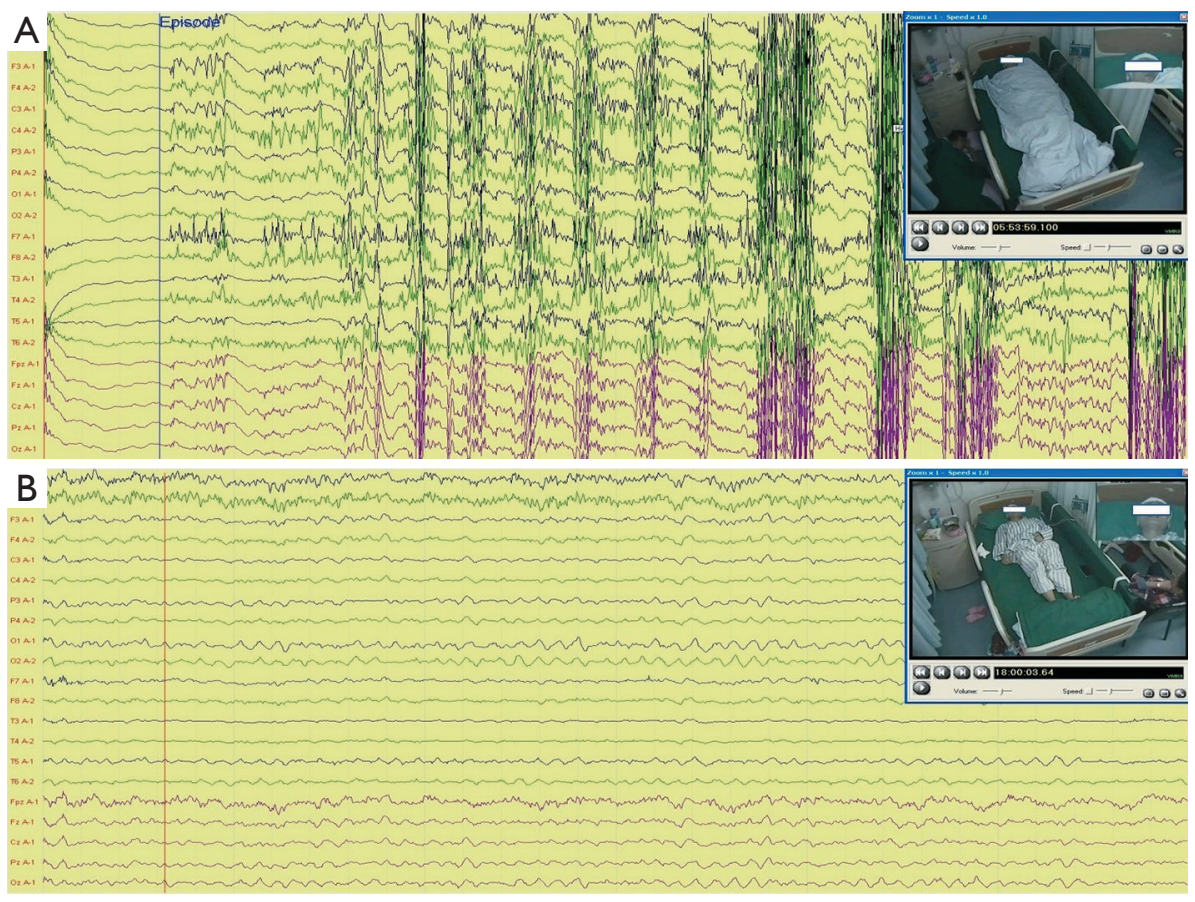

Figure 1 Video-EEG monitoring of ES (epileptic seizure) and PNES (psychogenic non-epileptic seizure). (A) ES: The patients experienced a sudden loss of consciousness, and oral and right limb automatism, followed by stiffening of the body (tonic phase) for 10-20 s and subsequent jerking of the extremities (clonic phase) for another 30-40 s. Low-voltage fast activity occurred and represented the initial ictal EEG pattern. (B) PNES: The patient reported indescribable anxiety and discomfort, then laid down on the bed, gradually developed repetitive head nodding followed by side-to-side head movement with preserved awareness. Eye closure and bilateral limb movement were also observed during the whole episode that lasted about $15 \mathrm{~min}$. Ictal epileptiform discharges were absent.

EEG confirmed co-concurrent PNES and ES in a middleaged woman. Right-sided anterior temporal lobectomy rendered the patient ES-free, and PNES also significantly decreased post operation without the need for additional psychiatric medication. We present the following case in accordance with the CARE reporting checklist (available at http://dx.doi.org/10.21037/atm-20-5423).

\section{Case presentation}

A right-handed 44-year-old woman was diagnosed with epilepsy at the age of 18 years, and over the next 25 years continued to experience multiple focal impaired awareness seizures (FIAS) every month, which mostly evolved into generalized tonic-clonic seizures (GTCS). The patient had a self-reported history of panic attack and anxiety disorder for 10 years, while she had no family history of epilepsy or related disorders. Having therapeutically failed on carbamazepine, phenobarbital and lamotrigine, the patient was referred to our epilepsy center for multi-disciplinary evaluation as a candidate for surgery. Her Hamilton Anxiety Rating Scale (HAM-A) score was 24 admission. Four-day continuous video-EEG monitoring captured three ES, all of right temporal origin (Figure 1A), and two PNES (Figure 1B). According to the patient and her family members, the three ES were typical seizures and two PNES were atypical in terms of semiology.

For ES revealed by video-EEG, the patient experienced a sudden loss of consciousness during sleep, followed by oral and right limb automatism, stiffening of body (tonic phase) for 10-20 s, and subsequent jerking of the extremities (clonic phase) for another 30-40 s. Low-voltage fast activity occurred on EEG and represented the initial ictal pattern. For PNES, the patient reported indescribable anxiety and discomfort, then gradually developed repetitive head nodding or side-to-side shaking with preserved consciousness. Eye closure and bilateral limb movement were also observed during the whole episode of PNES, which lasted for over $10 \mathrm{~min}$. Ictal epileptiform discharges 
were absent.

Magnetic resonance imaging (3T MRI) failed to show any positive findings. Hypometabolism in the right temporal lobe was seen on positron emission tomography (PET). Following presurgical evaluation including detailed history and clinical examination, scalp video-EEG monitoring, MRI, PET, and MEG (magnetoencephalography), the right anterior temporal lobectomy was successfully performed. Postoperative pathological examination revealed focal cortical dysplasia (FCD) type Ib in the resected tissue. The patient has been ES-free for one year until now. Her antiepileptic medication remained the same as prior to surgery. After surgery, the patient underwent no specific psychological or psychiatric treatment, and reported very occasional and mild PNES. Her HAM-A score decreased to 18 at the latest follow-up.

All procedures performed in studies involving human participants were in accordance with the ethical standards of the institutional research committee and with the Helsinki Declaration (as revised in 2013). Written informed consent was obtained from the patient.

\section{Discussion}

Over the past decades, resective surgery has been widely accepted as an important treatment option for medically refractory epilepsy $(8,9)$. However, investigations on whether surgical operation is favorable for some patients with simultaneous ES and PNES in terms of seizure control and life quality are limited. Some researchers proposed that PNES should be considered a relative contraindication for surgery (10). Our patient experienced significant benefits from anterior temporal lobectomy, which, in addition to other recent evidences (11), seems to suggest that the sole presence of either suspected or even video-EEG confirmed PNES may not be an absolute obstacle for epileptogenic zonectomy in some patients with comorbid intractable epilepsy. In other words, ES could have a similar chance of being controlled with surgical approach to those coconcurrent with PNES in certain patients.

Although primarily aimed at controlling ES, surgery for epilepsy also appears to be able to improve PNES symptoms in some cases. In 2002, Reuber et al. retrospectively reviewed the outcomes of 13 patients with PNES and ES who underwent epilepsy surgery. In their study, the ESs were originating from the temporal lobe in 10 cases. Post operation, nine patients were rid of PNES, two continued to have infrequent PNES, and the remaining two reported worsened PNES (12). Owing to the limited sample size in previous related studies, the overall surgical effects on PNES need to be further explored by a cohort study, which might vary according to the resected brain area and its relevant function. Given that PNES has strong psychosocial and psychiatric components, it would be interesting to further investigate the PNES outcomes with or without psychiatric medication following resection surgery, specifically involving the limbic system.

In addition, surgical control of ES might alter the habitual pattern of the patient, which could have untilnow unclarified influence on PNES and warrant future studies. Despite considerable improvement, our patient still reported infrequent episodes of PNES in the early postoperative period. We believe this patient needs longer follow-up and may require psychological and psychiatric intervention, as applicable. In the past 20 years, various forms of psychological interventions have been indicated to reduce the frequency of PNES and improve patients' psychosocial functioning, thereby optimizing the use of health service (13-15). Therefore, a multidisciplinary team comprising neurologist, neurosurgeon, psychologist, psychiatrist, and clinical neurophysiologist is crucial for the dual diagnosis and optimal management of patients with comorbid ES and PNES.

\section{Acknowledgments}

Funding: None.

\section{Footnote}

Reporting Checklist: The authors have completed the CARE reporting checklist. Available at http://dx.doi.org/10.21037/ atm-20-5423

Conflicts of Interest: All authors have completed the ICMJE uniform disclosure form (available at http://dx.doi. org/10.21037/atm-20-5423). The authors have no conflicts of interest to declare.

Ethical Statement: The authors are accountable for all aspects of the work in ensuring that questions related to the accuracy or integrity of any part of the work are appropriately investigated and resolved. All procedures performed in studies involving human participants were in accordance with the ethical standards of the institutional research committee and with the Helsinki Declaration (as 
revised in 2013). Written informed consent was obtained from the patient.

Open Access Statement: This is an Open Access article distributed in accordance with the Creative Commons Attribution-NonCommercial-NoDerivs 4.0 International License (CC BY-NC-ND 4.0), which permits the noncommercial replication and distribution of the article with the strict proviso that no changes or edits are made and the original work is properly cited (including links to both the formal publication through the relevant DOI and the license). See: https://creativecommons.org/licenses/by-nc-nd/4.0/.

\section{References}

1. Brown RJ, Syed TU, Benbadis S, et al. Psychogenic nonepileptic seizures. Epilepsy Behav 2011;22:85-93.

2. El-Naggar H, Moloney P, Widdess-Walsh P, et al. Simultaneous occurrence of nonepileptic and epileptic seizures during a single period of in-patient videoelectroencephalographic monitoring. Epilepsia Open 2017;2:467-71.

3. Gordon PC, Valiengo Lda C, Proença IC, et al. Comorbid epilepsy and psychogenic non-epileptic seizures: how well do patients and caregivers distinguish between the two. Seizure 2014;23:537-41.

4. Baroni G, Piccinini V, Martins WA, et al. Variables associated with co-existing epileptic and psychogenic nonepileptic seizures: a systematic review. Seizure 2016;37:35-40.

5. Smith BJ. Closing the Major Gap in PNES Research: Finding a Home for a Borderland Disorder. Epilepsy Curr 2014;14:63-7.

Cite this article as: Qiao L, Yu T, Li Y. Surgical outcome of a female patient with comorbid epilepsy and psychogenic nonepileptic seizures confirmed with video-EEG monitoring. Ann Transl Med 2021;9(3):273. doi: 10.21037/atm-20-5423
6. LaFrance WC Jr. Psychogenic nonepileptic "seizures" or "attacks"? It's not just semantics: seizures. Neurology 2010;75:87-8.

7. Stone J, LaFrance WC Jr, Brown R, et al. Conversion disorder: Current problems and potential solutions for DSM-5. J Psychosom Res 2011;71:369-76.

8. Jobst BC, Cascino GD. Resective epilepsy surgery for drug-resistant focal epilepsy: a review. JAMA 2015;313:285-93.

9. West S, Nolan SJ, Newton R. Surgery for epilepsy: a systematic review of current evidence. Epileptic Disord 2016;18:113-21.

10. Whitehead K, O'Sullivan S, Walker M. Impact of psychogenic nonepileptic seizures on epilepsy presurgical investigation and surgical outcomes. Epilepsy Behav 2015;46:246-8.

11. Furlan AER, da Silva SC Junior, Marques LHN, et al. Are psychogenic nonepileptic seizures risk factors for a worse outcome in patients with refractory mesial temporal epilepsy submitted to surgery? Results of a retrospective cohort study. Epilepsy Behav 2019;93:12-5.

12. Reuber M, Kurthen M, Fernández G, et al. Epilepsy surgery in patients with additional psychogenic seizures. Arch Neurol 2002;59:82-6.

13. LaFrance WC Jr, Reuber M, Goldstein LH. Management of psychogenic nonepileptic seizures. Epilepsia 2013;54:53-67.

14. Brown RJ, Reuber M. Psychological and psychiatric aspects of psychogenic nonepileptic seizures (PNES): a systematic review. Clin Psychol Rev 2016;45:157-82.

15. Carlson P, Perry KN. Psychological interventions for psychogenic non-epileptic seizures: a meta-analysis. Seizure 2017;45:142-50. 